\title{
CR automorphisms of real analytic manifolds in complex space
}

\author{
M.S. Baouendi ${ }^{1}$, P. EbenfelT ${ }^{2}$, And Linda Preiss Rothschild $^{3}$
}

\section{Introduction.}

In this paper we shall give sufficient conditions for local CR diffeomorphisms between two real analytic submanifolds of $\mathbb{C}^{N}$ to be determined by finitely many derivatives at finitely many points. These conditions will also be shown to be necessary in model cases. We shall also show that under the same conditions, the Lie algebra of the infinitesimal CR automorphisms at a point is finite dimensional.

Let $M$ be a real analytic submanifold of $\mathbb{C}^{N}$. For $p \in M$ a $C R$ vector at $p$ is a vector of the form $\sum_{j=1}^{N} c_{j} \frac{\partial}{\partial \bar{Z}_{j}}, c_{j} \in \mathbb{C}$, tangent to $M$ at $p$. If $M^{\prime}$ is another submanifold of $\mathbb{C}^{N}$, a mapping $F: M \rightarrow M^{\prime}$ is called $C R$ if for any $p \in M$ the pushforward $F_{*} X$ of any CR vector $X$ on $M$ at $p$ is a CR vector of $M^{\prime}$ at $F(p)$. In particular, the restriction to $M$ of a germ of a holomorphic diffeomorphism $H$ from $\mathbb{C}^{N}$ to itself is a CR map from $M$ to its image.

As in [BER1] (see Stanton [St1] for the case of a hypersurface), we shall say that a real submanifold of $\mathbb{C}^{N}$ is holomorphically nondegenerate if there is no germ of a nontrivial vector field $\sum_{j=1}^{N} c_{j}(Z) \frac{\partial}{\partial Z_{j}}$, with $c_{j}(Z)$ holomorphic, tangent to $M$. If $M$ is holomorphically nondegenerate there is an integer $l(M)$, with $0 \leq l(M)<N$, called the Levi number of $M$ (see $\S 1$ ) which measures the holomorphic nondegeneracy of $M$. If $M$ is a Levi-nondegenerate hypersurface then $l(M)=1$. A connected real analytic submanifold is minimal almost everywhere if there is no germ of a holomorphic function whose restriction to $M$ is a nonconstant real-valued function. This coincides with the notion of being minimal at most points in the sense of Tumanov [Tu1].

\footnotetext{
${ }^{1}$ Partially supported by National Science Foundation Grant DMS 95-01516.

${ }^{2}$ Supported by a grant from the Swedish Natural Science Research Council.

${ }^{3}$ Partially supported by National Science Foundation Grant DMS 95-01516.
} 
If $M$ is a hypersurface which is holomorphically nondegenerate, then $M$ is minimal almost everywhere.

The following uniqueness result is one of the main theorems of this paper.

Theorem 1. Let $M \subset \mathbb{C}^{N}$ be a connected, real analytic, holomorphically nondegenerate submanifold of codimension $d$ and Levi number $l(M)$ such that $M$ is minimal almost everywhere. Then for all $p \in M$ outside a proper real analytic subvariety of $M$ the following holds. If $M^{\prime} \subset \mathbb{C}^{N}$ is another real analytic submanifold with $\operatorname{dim}_{\mathbb{R}} M^{\prime}=\operatorname{dim}_{\mathbb{R}} M$, and $F, G$ are smooth germs at $p$ of $C R$ diffeomorphisms of $M$ into $M^{\prime}$ such that in some local coordinates $x$ on $M$

$$
\frac{\partial^{|\alpha|} F}{\partial x^{\alpha}}(p)=\frac{\partial^{|\alpha|} G}{\partial x^{\alpha}}(p)
$$

for all $|\alpha| \leq(d+1) l(M)$, then $F \equiv G$.

Corollary. Let $M$ be as in Theorem 1. Then for all $p \in M$ outside a proper real analytic subvariety of $M$ the following holds. If $H$ is a germ at $p$ of a local biholomorphism of $\mathbb{C}^{N}$ mapping $M$ into itself and fixing $p$, with

$$
\frac{\partial H_{j}}{\partial Z_{k}}(p)=\delta_{j k}, \quad \frac{\partial^{|\alpha|} H_{j}}{\partial Z^{\alpha}}(p)=0,1 \leq j, k \leq N, 2 \leq|\alpha| \leq(d+1) l(M),
$$

then $H$ is the identity map on $M$.

In fact, Theorem 1 also follows from the statement of the Corollary. In case $M$ is a Levi-nondegenerate hypersurface (i.e. $d=1$ and $l(M)=1$ ) Theorem 1 reduces to the result of Chern-Moser [CM] that a germ of a CR diffeomorphism is uniquely determined by its derivatives of order $\leq 2$ at a point. Generalizations of this result for Levi nondegenerate manifolds of higher codimension were later given by Tumanov-Henkin [TH], Tumanov [Tu2]. More precise results for Levi nondegenerate hypersurfaces have been given by Beloshapka [Be] and Loboda [L].

A smooth real vector field $X$ defined in a neighborhood of $p$ in $M$ is an infinitesimal holomorphism if the local 1-parameter group of diffeomorphisms $\exp t X$ for $t$ small extend to a local 1-parameter group of biholomorphisms of $\mathbb{C}^{N}$. More generally, $X$ is called an infinitesimal $C R$ automorphism if the exp $t X$ are CR diffeomorphisms. We denote by $\operatorname{hol}(M, p)$ $($ resp. aut $(M, p))$ the Lie algebra generated by the infinitesimal holomorphisms (resp. infinitesimal CR automorphisms). Since every local biholomorphism preserving $M$ restricts to a CR diffeomorphism of $M$ into itself, it 
follows that $\operatorname{hol}(M, p) \subset \operatorname{aut}(M, p)$. It follows from the work of Tanaka [Ta] that $\operatorname{hol}(M, p)$ is a finite dimensional vector space if $M$ is a real analytic Levi nondegenerate hypersurface. Recently Stanton [St2] proved that if $M$ is a real analytic hypersurface, $\operatorname{hol}(M, p)$ is a finite dimensional real vector space for any $p \in M$ if and only if $M$ is holomorphically nondegenerate. In this paper we prove more general results for any real analytic CR submanifold. Recall that a real analytic submanifold $M$ of $\mathbb{C}^{N}$ of codimension $d$ is $C R$ if $M$ is locally defined by the vanishing of $d$ real valued real analytic functions $\rho_{1}, \ldots, \rho_{d}$, with linearly independent differentials, such that the linear span of the complex differentials $\partial \rho_{1}, \ldots, \partial \rho_{d}$ is of constant dimension.

Theorem 2. Let $M \subset \mathbb{C}^{N}$ be a real analytic, connected $C R$ submanifold. If $M$ is holomorphically nondegenerate, and minimal almost everywhere then

$$
\operatorname{dim}_{\mathbb{R}} \operatorname{aut}(M, p)<\infty
$$

for all $p \in M$.

Theorems 1 and 2 are optimal in the sense that holomorphic nondegeneracy is necessary for the conclusions of Theorems 1 and 2 and that the condition that $M$ is minimal almost everywhere is necessary in model cases. We have the following result.

Theorem 3. Let $M \subset \mathbb{C}^{N}$ be a connected real analytic $C R$ submanifold.

(i) If $M$ is holomorphically degenerate, then for any $p \in M$ and any integer $K>0$ there exist local biholomorphisms $F$ and $G$ near $p$ mapping $M$ into itself and fixing $p$ such that

$$
\frac{\partial^{|\alpha|} F}{\partial Z^{\alpha}}(p)=\frac{\partial^{|\alpha|} G}{\partial Z^{\alpha}}(p)
$$

for all $|\alpha| \leq K$, but $F \not \equiv G$ on $M$. Furthermore, $\operatorname{dim}_{\mathbb{R}} \operatorname{hol}(M, p)=\infty$.

(ii) If $M$ is holomorphically nondegenerate but nowhere minimal then for $p$ in an open dense set in $M$ either $\operatorname{dim}_{\mathbb{R}} \operatorname{hol}(M, p)=\infty$ or $\operatorname{dim}_{\mathbb{R}} \operatorname{hol}(M, p)=0$.

(iii) If $M$ is defined by the vanishing of weighted homogeneous polynomials, and nowhere minimal then for any $p \in M$ and any integer $K>0$ there exist local biholomorphisms $F$ and $G$ near $p$ mapping $M$ into itself and fixing $p$ such that (0.4) holds for all $|\alpha| \leq K$, but $F \not \equiv G$ on $M$. Also $\operatorname{dim}_{\mathbb{R}} \operatorname{hol}(M, p)=\infty$. 
The paper is organized as follows. In $\S 1$ we recall some results about holomorphically nongenerate manifolds and Segre sets of a generic manifold. In $\S 2$ we prove a result on uniqueness of CR diffeomorphisms, of which Theorem 1 is a consequence. In $\S 3$ we study infinitesimal CR automorphisms and prove Theorem 2. In $\S 4$ we prove Theorem 3 and also construct an example to show that one can have $\operatorname{dim}_{\mathbb{R}} \operatorname{hol}(M, p)=0$ even if $M$ is nowhere minimal. In $\S 5$ we make some remarks concerning the group $G_{p}$ of local biholomorphisms leaving $M$ invariant and fixing a point $p \in M$.

\section{Preliminaries.}

\subsection{Holomorphic nondegeneracy and $k$-nondegeneracy of real analytic CR submanifolds.}

Let $M \subset \mathbb{C}^{N}$ be a connected real analytic CR submanifold. We denote by $\mathcal{V}$ the largest holomorphic submanifold in $\mathbb{C}^{N}$ containing $M$ with minimum dimension. As in [BER1] we call $\mathcal{V}$ the intrinsic complexification of $M$. If $\mathcal{V}$ is all of $\mathbb{C}^{N}$, then $M$ is called generic. Thus any CR submanifold is generic when considered as a submanifold of its intrinsic complexification. Recall that $M$ is called holomorphically degenerate at $p_{0} \in M$ if there is a germ of a holomorphic vector field at $p_{0}$ which is tangent to $M$ but not trivial (i.e. not identically 0 ) on $M$. It can be easily checked that $M$ is holomorphically degenerate at $p_{0}$ as a submanifold of $\mathbb{C}^{N}$ if and only if it is holomorphically degenerate at $p_{0}$ as a submanifold of $\mathcal{V}$.

We say that $M$ is holomorphically nondegenerate if it is not holomorphically degenerate at any point. In fact it is proved in [BER1] that if $M$ is holomorphically degenerate at one point, it is holomorphically degenerate at every point.

Suppose $M$ is a real analytic generic submanifold of $\mathbb{C}^{N}$ and that $M$ is defined near $p_{0} \in M$ by $\rho(Z, \bar{Z})=0$, where $\rho=\left(\rho_{1}, \ldots, \rho_{d}\right)$ are real valued real analytic functions with $\rho\left(p_{0}, \bar{p}_{0}\right)=0$ and $\partial \rho_{1} \wedge \ldots \wedge \partial \rho_{d} \neq 0$ near $p_{0}$. Let $L=\left(L_{1}, \ldots, L_{n}\right)$ be a basis for the CR vector fields on $M$ near $p_{0}$. For any multi-index $\alpha$ put $L^{\alpha}=L_{1}^{\alpha_{1}} \ldots L_{n}^{\alpha_{n}}$. Introduce, for $j=1, \ldots, d$ and any multi-index $\alpha$, the vectors

$$
V_{j \alpha}(Z, \bar{Z})=L^{\alpha} \rho_{j Z}(Z, \bar{Z})
$$

where $\rho_{j Z}$ denotes the gradient of $\rho_{j}$ with respect to $Z$. We say that the generic real analytic submanifold $M$ is $k$-nondegenerate at $p_{0}$ if $k$ is the 
smallest positive integer for which the span of the vectors $V_{j \alpha}\left(p_{0}, \bar{p}_{0}\right)$, for $j=1, \ldots, d$ and $|\alpha| \leq k$, equals $\mathbb{C}^{N}$. This definition is independent of the coordinate system used, the defining equations of $M$, and the choice of basis $L$. We say that a real analytic CR submanifold $M$ is $k$-nondegenerate at $p_{0} \in M$ if $M$ is $k$-nondegenerate at $p_{0}$ as a generic submanifold of its intrinsic complexification $\mathcal{V}$.

If $M$ is a connected $\mathrm{CR}$ submanifold of $\mathbb{C}^{N}$ and $\mathcal{V}$ its intrinsic complexification, then the $C R$ dimension of $M$ is the nonnegative integer defined by

$$
\operatorname{dim}_{\mathbb{R}} M=\operatorname{dim}_{\mathbb{C}} \mathcal{V}+C R \operatorname{dim} M
$$

The following proposition is in [BER1, Proposition 1.3.1] for generic manifolds. Its extension to CR manifolds is immediate.

Proposition 1.1.1. Let $M \subset \mathbb{C}^{N}$ be a connected real analytic $C R$ submanifold of CR dimension $n$. Then the following are equivalent.

(i) $M$ is holomorphically nondegenerate.

(ii) There exists $p_{1} \in M$ and $k>0$ such that $M$ is $k$-nondegenerate at $p_{1}$.

(iii) There exists $V$, a proper real analytic subset of $M$, and an integer $l=l(M), 0 \leq l(M) \leq n$, such that $M$ is l-nondegenerate at every $p \in M \backslash V$.

The number $l(M)$ given in (iii) is called the Levi number of $M$.

\subsection{The Segre sets.}

In this section, we introduce the Segre sets of a generic real analytic submanifold in $\mathbb{C}^{N}$ and recall some of their properties. We refer the reader to the paper [BER1] for a more detailed account (including proofs of the main results) of these sets. Let $M$ denote a generic real analytic submanifold in some neighborhood $U \subset \mathbb{C}^{N}$ of $p_{0} \in M$. Let $\rho=\left(\rho_{1}, \ldots \rho_{d}\right)$ be defining functions as above, and choose holomorphic coordinates $Z=\left(Z_{1}, \ldots, Z_{N}\right)$ vanishing at $p_{0}$. Embed $\mathbb{C}^{N}$ in $\mathbb{C}^{2 N}=\mathbb{C}_{Z}^{N} \times \mathbb{C}_{\zeta}^{N}$ as the real plane $\{(Z, \zeta) \in$ $\left.\mathbb{C}^{2 N} \zeta=\bar{Z}\right\}$. Let us denote by $\operatorname{pr}_{Z}$ and $\operatorname{pr}_{\zeta}$ the projections of $\mathbb{C}^{2 N}$ onto $\mathbb{C}_{Z}^{N}$ and $\mathbb{C}_{\zeta}^{N}$, respectively. The natural anti-holomorphic involution $\sharp$ in $\mathbb{C}^{2 N}$ defined by

$$
\sharp(Z, \zeta)=(\bar{\zeta}, \bar{Z})
$$


leaves the plane $\{(Z, \zeta) \zeta=\bar{Z}\}$ invariant. This involution induces the usual anti-holomorphic involution in $\mathbb{C}^{N}$ by

$$
\mathbb{C}^{N} \ni Z \mapsto \operatorname{pr}_{\zeta}\left({ }^{\sharp} \operatorname{pr}_{Z}^{-1}(Z)\right)=\bar{Z} \in \mathbb{C}^{N} .
$$

Given a set $S$ in $\mathbb{C}_{Z}^{N}$ we denote by ${ }^{*} S$ the set in $\mathbb{C}_{\zeta}^{N}$ defined by

$$
{ }^{*} S=\operatorname{pr}_{\zeta}\left({ }^{\sharp} \operatorname{pr}_{Z}^{-1}(S)\right)=\{\zeta \bar{\zeta} \in S\} .
$$

By a slight abuse of notation, we use the same notation for the corresponding transformation taking sets in $\mathbb{C}_{\zeta}^{N}$ to sets in $\mathbb{C}_{Z}^{N}$. Note that if $X$ is a complex analytic set defined near $Z^{0}$ in some domain $\Omega \subset \mathbb{C}_{Z}^{N}$ by $h_{1}(Z)=\ldots=$ $h_{k}(Z)=0$, then ${ }^{*} X$ is the complex analytic set in ${ }^{*} \Omega \subset \mathbb{C}_{\zeta}^{N}$ defined near $\zeta^{0}=\bar{Z}^{0}$ by $\bar{h}_{1}(\zeta)=\ldots=\bar{h}_{k}(\zeta)=0$. Here, given a holomorphic function $h(Z)$ we use the notation $\bar{h}(Z)=\overline{h(\bar{Z})}$.

Denote by $\mathcal{M} \subset \mathbb{C}^{2 N}$ the complexification of $M$ given by

$$
\mathcal{M}=\left\{(Z, \zeta) \in \mathbb{C}^{2 N} \rho(Z, \zeta)=0\right\} .
$$

This is a complex submanifold of codimension $d$ in some neighborhood of 0 in $\mathbb{C}^{2 N}$. We choose our neighborhood $U$ in $\mathbb{C}^{N}$ so small that $U \times{ }^{*} U \subset \mathbb{C}^{2 N}$ is contained in the neighborhood where $\mathcal{M}$ is a manifold. Note that $\mathcal{M}$ is invariant under the involution $\sharp$ defined in (1.2.1).

We associate to $M$ at $p_{0}$ a sequence of germs of sets $N_{0}, N_{1}, \ldots, N_{j_{0}}$ at $p_{0}$ in $\mathbb{C}^{N}$-the Segre sets of $M$ at $p_{0}$-defined as follows. Put $N_{0}=\left\{p_{0}\right\}$ and define the consecutive sets inductively (the number $j_{0}$ will be defined later) by

$$
N_{j+1}=\operatorname{pr}_{Z}\left(\mathcal{M} \cap \operatorname{pr}_{\zeta}^{-1}\left({ }^{*} N_{j}\right)\right)=\operatorname{pr}_{Z}\left(\mathcal{M} \cap{ }^{\sharp} \operatorname{pr}_{Z}^{-1}\left(N_{j}\right)\right) .
$$

Here, and in what follows, we identify a germ $N_{j}$ with some representative of it. These sets are, by definition, invariantly defined and they arise naturally in the study of mappings between submanifolds (see later sections in this paper, and [BER1]).

The sets $N_{j}$ can be described in terms of the defining equations $\rho(Z, \bar{Z})=$ 0 (see [BER1, §2.2]), e.g.

$$
N_{1}=\{Z \rho(Z, 0)=0\}
$$

and

$$
N_{2}=\left\{Z \exists \zeta^{1} \rho\left(Z, \zeta^{1}\right)=0, \rho\left(0, \zeta^{1}\right)=0\right\}
$$


We have the inclusions

$$
N_{0} \subset N_{1} \subset \ldots \subset N_{j} \subset \ldots
$$

and $j_{0}$ is the largest number such that the first $j_{0}$ inclusions in (1.2.8) are strict. (The Segre sets stabilize after that, and $N_{j_{0}+1}=N_{j_{0}+2}=\ldots$.) It is shown in [BER1] that, in suitable coordinates $(z, w) \in \mathbb{C}^{n} \times \mathbb{C}^{d}=\mathbb{C}^{N}$ (so-called normal coordinates), the Segre set $N_{j}$, for $j=1, \ldots, j_{0}$, can also be defined as images of certain holomorphic mappings

$$
\mathbb{C}^{n} \times \mathbb{C}^{(j-1) n} \ni(z, \Lambda) \mapsto\left(z, v^{j}(z, \Lambda)\right) \in \mathbb{C}^{N} .
$$

Thus, we can define the generic dimension $d_{j}$ of $N_{j}$ as the generic rank of the mapping (1.2.9).

So far we have only considered generic submanifolds. If $M$ is a real analytic CR submanifold of $\mathbb{C}^{N}$, then $M$ is generic as a submanifold of its intrinsic complexification $\mathcal{V}$ (see $\S 1.1$ ). The Segre sets of $M$ at a point $p_{0} \in M$ can be defined as subsets of $\mathbb{C}^{N}$ by the process described at the beginning of this subsection (i.e. by (1.2.5)) just as for generic submanifolds or they can be defined as subsets of $\mathcal{V}$ by identifying $\mathcal{V}$ near $p_{0}$ with $\mathbb{C}^{K}$ and considering $M$ as a generic submanifold of $\mathbb{C}^{K}$. It can be shown that these definitions are equivalent.

The main properties concerning the Segre sets that we shall use in this paper are summarized in the following theorem.

Theorem 1.2.1. Let $M$ be a real analytic CR submanifold in $\mathbb{C}^{N}$, and let $p_{0} \in M$.

(a) Denote by $W$ the $C R$ orbit of $p_{0}$ (i.e. the Nagano leaf or, equivalently, the CR submanifold of $M$ of smallest dimension, with the same $C R$ dimension as $M$, through $p_{0}$ ) and by $X$ its intrinsic complexification (see §1.1). Then the maximal Segre set $N_{j_{0}}$ of $M$ at $p_{0}$ is contained in $X$ and $N_{j_{0}}$ contains an open subset of $X$, i.e. $d_{j_{0}}=\operatorname{dim}_{\mathbb{C}} X$.

(b) There are holomorphic immersions $Z_{0}\left(t_{0}\right), Z_{1}\left(t_{1}\right), \ldots, Z_{j_{0}}\left(t_{j_{0}}\right)$ defined near the origin,

$$
\mathbb{C}^{d_{j}} \ni t_{j} \mapsto Z_{j}\left(t_{j}\right) \in \mathbb{C}^{N},
$$

and holomorphic maps $s_{0}\left(t_{1}\right), \ldots, s_{j_{0}-1}\left(t_{j_{0}}\right)$,

$$
\mathbb{C}^{d_{j}} \ni t_{j} \mapsto s_{j-1}\left(t_{j}\right) \in \mathbb{C}^{d_{j-1}},
$$


such that $Z_{j}\left(t_{j}\right)$ has rank $d_{j}$ near the origin, $Z_{j}\left(t_{j}\right) \in N_{j}$, and such that

$$
\left(Z_{j}\left(t_{j}\right), \bar{Z}_{j-1}\left(s_{j-1}\left(t_{j}\right)\right)\right) \in \mathcal{M}
$$

for $j=1, \ldots, j_{0}$.

Proof. Part (a) is contained in [BER1, Theorem 2.2.1], and the mappings in part (b) are constructed in the paragraph following [BER1, Assertion 3.3.2].

Remark 1.2.2.. The holomorphic immersion $Z_{j}\left(t_{j}\right), j=0,1, \ldots, j_{0}$, in part (b) above provides a parametrization of an open piece of $N_{j}$. However, this piece of $N_{j}$ need not contain the point $p_{0}$. Indeed, $N_{j}$ need not even be a manifold at $p_{0}$.

Recall that a CR submanifold $M$ is said to be minimal at a point $p_{0} \in M$ if there is no proper CR submanifold of $M$ through $p_{0}$ with the same CR dimension as $M$. For a real analytic submanifold, this notion coincides with the notion of finite type in the sense of Bloom-Graham [BG]. One can check that if $M$ is connected then $M$ is minimal almost everywhere, as defined in $\S 0$ if and only if $M$ is minimal at some point in the sense described above (see e.g. [BER1, Lemma3.4.1]). The following is an immediate consequence of the theorem.

Corollary 1.2.3. Let $M$ be a real analytic generic submanifold in $\mathbb{C}^{N}$ and $p_{0} \in M$. Then $M$ is minimal at $p_{0}$ if and only if $d_{j_{0}}=N$ or, equivalently, if and only if the maximal Segre set at $p_{0}$ contains an open subset of $\mathbb{C}^{N}$.

\section{Uniqueness of CR diffeomorphisms.}

The main result here is the following, which implies Theorem 1 as a special case.

Theorem 2.1. Let $M \subset \mathbb{C}^{N}$ be a connected real analytic, holomorphically nondegenerate $C R$ submanifold and let $d$ be the (real) codimension of $M$ in its intrinsic complexificiation. Suppose that there is a point $p \in M$ at which $M$ is minimal. For any $p_{0} \in M$ there exists a finite set of points $p_{1}, \ldots, p_{k} \in M$ such that if $M^{\prime} \subset \mathbb{C}^{N}$ is another real analytic $C R$ submanifold 
with $\operatorname{dim}_{\mathbb{R}} M^{\prime}=\operatorname{dim}_{\mathbb{R}} M$, and $F, G$ are smooth $C R$ diffeomorphisms of $M$ into $M^{\prime}$ such that

$$
\frac{\partial^{|\alpha|} F}{\partial x^{\alpha}}\left(p_{l}\right)=\frac{\partial^{|\alpha|} G}{\partial x^{\alpha}}\left(p_{l}\right)
$$

for $l=1, \ldots, k$, and $|\alpha| \leq(d+1) l(M)$, then $F \equiv G$ in a neighborhood of $p_{0}$ in $M$. If $M$ is minimal at $p_{0}$, then one can take $k=1$. If, in addition, $M$ is $l(M)$-nondegenerate at $p_{0}$, then one may take $p_{1}=p_{0}$.

\section{Remarks.}

(i) The condition (2.1) can be expressed by saying that the $(d+1) l(M)$ jets of the mappings coincide at all the points $p_{1}, \ldots, p_{k}$.

(ii) The choice of points $p_{1}, \ldots, p_{k}$ can be described as follows. Let $U_{1}, \ldots, U_{k}$ be the components of the set of minimal points of $M$ in $U$, a sufficiently small neighborhood of $p_{0}$ in $M$, which have $p_{0}$ in their closure. For each $l=1, \ldots, k$, we may choose any $p_{l}$ from the dense open subset of $U_{l}$ consisting of those points which are $l(M)$-nondegenerate.

Before we prove Theorem 2.1 we need some preliminary results.

Proposition 2.2. Let $M, M^{\prime} \subset \mathbb{C}^{N}$ be real analytic CR submanifolds, and $p_{0} \in M$. Assume that $M$ is holomorphically nondegenerate and generic, and that $M$ is $l(M)$-nondegenerate at $p_{0}$. Let $H$ be a germ of a biholomorphism of $\mathbb{C}^{N}$ at $p_{0}$ such that $H(M) \subset M^{\prime}$. Then there are $\mathbb{C}^{N}$ valued functions $\Psi^{\gamma}$, holomorphic in all of their arguments, such that

$$
\frac{\partial^{|\gamma|} H}{\partial Z^{\gamma}}(Z)=\Psi^{\gamma}\left(Z, \zeta, \bar{H}(\zeta), \ldots, \frac{\partial^{|\alpha|} \bar{H}}{\partial \zeta^{\alpha}}(\zeta), \ldots\right),
$$

where $|\alpha| \leq l(M)+|\gamma|$, for all multi-indices $\gamma$ and all points $(Z, \zeta) \in \mathcal{M}$ near $\left(p_{0}, \bar{p}_{0}\right)$. Moreover, the functions $\Psi^{\gamma}$ depend only on $M, M^{\prime}$ and

$$
\frac{\partial^{|\beta|} H}{\partial Z^{\beta}}\left(p_{0}\right), \quad|\beta| \leq l(M) .
$$

Proof. It suffices to prove (2.2) in any coordinate system of the target space near $p_{0}^{\prime}=H\left(p_{0}\right)$. If we choose normal coordinates for $M^{\prime}$ at $p_{0}^{\prime}$ then the proof is exactly the same as the proof of Assertion 3.3.1 and subsequent remarks in [BER1] (see also [BR1, Lemma 2.3]). 
Proposition 2.3. Let $M \subset \mathbb{C}^{N}$ be a real analytic, holomorphically nondegenerate $C R$ submanifold and $p_{0} \in M$. Let $X$ be the intrinsic complexification of the $C R$ orbit $W$ of $p_{0}$ in $M$ and $d$ the codimension of $W$ in $X$. Assume that $M$ is $l(M)$-nondegenerate at $p_{0}$. If $H^{1}, H^{2}$ are germs of biholomorphisms of $\mathbb{C}^{N}$ at $p_{0}$ such that $H^{1}(M), H^{2}(M) \subset M^{\prime}$, where $M^{\prime}$ is another real analytic CR submanifold with $\operatorname{dim}_{\mathbb{R}} M^{\prime}=\operatorname{dim}_{\mathbb{R}} M$, and such that

$$
\frac{\partial^{|\alpha|} H^{1}}{\partial Z^{\alpha}}\left(p_{0}\right)=\frac{\partial^{|\alpha|} H^{2}}{\partial Z^{\alpha}}\left(p_{0}\right), \quad|\alpha| \leq(d+1) l(M),
$$

then $\left.\left.H^{1}\right|_{X} \equiv H^{2}\right|_{X}$.

Proof. The intrinsic complexification of the CR orbit is contained in the intrinsic complexification of $M$, and the notion of holomorphic nondegeneracy is independent of the ambient space. Similarly, the notion of $l(M)$ nondegeneracy is defined in the intrinsic complexification. Hence we may reduce to the case where $M$ is generic; we shall assume this for the rest of the proof.

Let $N_{j}, j=0,1, \ldots, j_{0}$, be the Segre sets of $M$ at $p_{0}$, and let $Z_{0}\left(t_{0}\right), \ldots$, $Z_{j_{0}}\left(t_{j_{0}}\right)$ be the canonical parametrizations of the $N_{j}$ 's and $s_{0}\left(t_{1}\right), \ldots, s_{j_{0}-1}$ $\left(t_{j_{0}}\right)$ the associated maps so that

$$
\left(Z_{j+1}\left(t_{j+1}\right), \bar{Z}_{j}\left(s_{j}\left(t_{j+1}\right)\right)\right) \in \mathcal{M},
$$

for all $j=0, \ldots, j_{0}-1$ (see Theorem 1.2.1 (b)). In view of (2.4) and Proposition 2.2, there are functions $\Psi^{\gamma}$ such that both $H^{1}$ and $H^{2}$ satisfy the identity $(2.2)$ for $(Z, \zeta) \in \mathcal{M}$. Substituting (2.5) with $j=0$ into this identity and recalling that $Z_{0}\left(t_{0}\right) \equiv p_{0}$ (i.e. it is the constant map), we deduce that $H^{1}$ and $H^{2}$ as well as all their derivatives are identical on the first Segre set $N_{1}$. Note that since each $N_{j}$ is the holomorphic image of a connected set, if two holomorphic functions agree on an open piece, they agree on all of $N_{j}$. By inductively substituting (2.5) into (2.2) for $j=1, \ldots, j_{0}-1$, we deduce that the restrictions of the mappings $H^{1}$ and $H^{2}$, as well as all their derivatives, to the maximal Segre set $N_{j_{0}}$ are identical. The conclusion of the proposition now follows from Theorem 2.2.1 (a), since $N_{j_{0}}$ contains an open piece of $X$.

We now proceed with the proof of Theorem 2.1.

Proof of Theorem 2.1. By a change of holomorphic coordinates near $p_{0}$ and by shrinking $M$, if necessary, we may assume that $M$ is a real analytic, 
holomorphically nondegenerate, generic submanifold of $\mathbb{C}^{K}$, for some $K \leq$ $N$.

Let $V$ be the set of points on $M$ at which $M$ is not minimal. This is a real analytic subset and, since $M$ is minimal at some point $p, V$ is also proper. Denote by $U_{1}, \ldots, U_{k}$ those components of $M \backslash V$ that have $p_{0}$ in their closure. Clearly, $k$ is a finite number since $M \backslash V$ is a semianalytic subset (a semi-analytic set is locally finite in the sense that only a finite number of components meet each compact set). Also, since $M$ is holomorphically nondegenerate, $M$ is $l(M)$-nondegenerate outside a proper real analytic subset. Pick $p_{l} \in U_{l}$, for $l=1, \ldots, k$, such that $M$ is $l(M)$ nondegenerate at $p_{l}$.

Since $M$ is minimal in $U_{l}$, it follows from a result of Tumanov [Tu1] that for every compact set $K_{l} \subset U_{l}$ both $F$ and $G$ extend holomorphically into an open connected wedge $\Omega_{l}$ in $\mathbb{C}^{K}$ with edge on $K_{l}$. Also, since $M^{\prime}$ is CR diffeomorphic with $M$, it follows that the intrinsic complexification $\mathcal{V}^{\prime}$ of $M^{\prime}$ has complex dimension $K$ as well. Since $M$ is $l(M)$-nondegenerate at $p_{l}$ and hence also essentially finite at $p_{l}$ (see [BER1]), it follows that both $F$ and $G$ extend as biholomorphisms of some neighborhood of $p_{l}$ in $\mathbb{C}^{K}$ onto a neighborhood of $F\left(p_{l}\right) \in M^{\prime}$ in $\mathcal{V}^{\prime}$ (see [BJT]). It follows from (2.1) and Proposition 2.3 that the holomorphic extensions of $F$ and $G$ into $\Omega_{l}$ are identical. Consequently, $F \equiv G$ in $U_{l}$ and, since this is true for any $l=1, \ldots, k$, the theorem follows by continuity of the mappings.

\section{The infinitesimal CR automorphisms.}

\subsection{The minimal case.}

We shall prove Theorem 2 in this section. Let $X^{1}, \ldots, X^{m} \in \operatorname{aut}\left(M, p_{0}\right)$ be linearly independent over $\mathbb{R}$. Let $x=\left(x_{1}, \ldots, x_{r}\right)$ be a local coordinate system on $M$ vanishing at $p_{0}$. In this coordinate system, we may write

$$
X^{j}=\sum_{l=1}^{r} \tilde{X}_{l}^{j}(x) \frac{\partial}{\partial x_{l}}=\tilde{X}^{j}(x) \cdot \frac{\partial}{\partial x} .
$$

For $y=\left(y_{1}, \ldots, y_{m}\right) \in \mathbb{R}^{m}$, we denote by $\Phi(t, x, y)$ the flow of the vector field $y_{1} X_{1}+\ldots+y_{m} X_{m}$, i.e. the solution of

$$
\left\{\begin{array}{l}
\frac{\partial \Phi}{\partial t}(t, x, y)=\sum_{i=1}^{m} y_{i} \tilde{X}^{i}(\Phi(t, x, y)) \\
\Phi(0, x, y)=x
\end{array}\right.
$$


By choosing $\delta>0$ sufficiently small, there is $c>0$ such that the flows $\Phi(t, x, y)$ are smooth $\left(C^{\infty}\right)$ in $\left\{(t, x, y) \in \mathbb{R}^{1+r+m}|t| \leq 2,|x| \leq c,|y| \leq \delta\right\}$. This follows from the identity

$$
\Phi(s t, x, y)=\Phi(t, x, s y), \quad s \in \mathbb{R},
$$

which, in turn, follows from the fact that the solution of (3.1.2) is unique (the reader can verify that the left side of (3.1.3) solves the initial value problem (3.1.2) that defines the right side of (3.1.3)). Denote by $F(x, y)$ the corresponding time-one maps, i.e.

$$
F(x, y)=\Phi(1, x, y) .
$$

Assertion 3.1.1. There is a $\delta^{\prime}, 0<\delta^{\prime}<\delta$, such that for any fixed $y_{1}, y_{2}$ with $\left|y^{1}\right|,\left|y^{2}\right| \leq \delta^{\prime}$, if $F\left(x, y^{1}\right) \equiv F\left(x, y^{2}\right)$ for $|x| \leq c$ then necessarily $y^{1}=$ $y^{2}$.

Proof of Assertion 3.1.1. Note from (3.1.3) that, with the notation $e_{i}$ for $i$ th unit vector in $\mathbb{R}^{m}$,

$$
\frac{\partial F}{\partial y_{i}}(x, 0)=\frac{d}{d s}\left[\Phi\left(1, x, s e_{i}\right)\right]_{s=0}=\frac{\partial \Phi}{\partial t}\left(0, x, e_{i}\right)=\tilde{X}^{i}(x) .
$$

Thus, denoting by $\tilde{X}(x)$ the $r \times m$-matrix with column vectors $\tilde{X}^{i}(x)$, we have

$$
\frac{\partial F}{\partial y}(x, 0)=\tilde{X}(x)
$$

If $J(x)=\left(J_{1}(x), \ldots, J_{r}(x)\right)$ is a continuous mapping into $\mathbb{R}^{r}$ for $|x| \leq c$ then we put

$$
\|J(x)\|=\sup _{|x| \leq c}\left(\sum_{l=1}^{r} J_{l}(x)^{2}\right)^{1 / 2}
$$

By Taylor expansion we obtain

$$
\left\|F\left(x, y^{2}\right)-F\left(x, y^{1}\right)\right\| \geq\left\|\frac{\partial F}{\partial y}\left(x, y^{1}\right) \cdot\left(y^{2}-y^{1}\right)\right\|-C\left|y^{2}-y^{1}\right|^{2}
$$

where $C>0$ is some uniform constant for $\left|y^{1}\right|,\left|y^{2}\right| \leq \delta$. Now, by assumption, the vector fields $X^{1}, \ldots, X^{m}$ are linearly independent over $\mathbb{R}$. This means precisely that there is a constant $C^{\prime}$ such that

$$
\|\tilde{X}(x) \cdot y\| \geq C^{\prime}|y| .
$$


Using (3.1.6), (3.1.9), the smoothness of $F$, and a standard compactness argument, we deduce from (3.1.8) that the conclusion of Assertion 3.1.1 holds.

Now, we proceed with the proof of Theorem 2. Denote by $U$ the open neighborhood of $p$ on $M$ given by $|x|<c$. We make use of Theorem 2.1 with $M$ replaced by $U$. Let $p_{1}, \ldots, p_{k}$ be the points in $U$ given by the theorem. By choosing the number $\delta^{\prime}>0$ in Assertion 3.1.1 even smaller if necessary, we may assume that the maps $x \mapsto F(x, y)$, for $|y|<\delta^{\prime}$, are CR diffeomorphisms of $U$ into $M$. Consider the smooth mapping from $|y|<\delta^{\prime}$ into $\mathbb{R}^{\mu}$ defined by

$$
y \mapsto\left(\frac{\partial^{|\alpha|} F\left(p_{l}, y\right)}{\partial x^{\alpha}}\right) \in \mathbb{R}^{\mu},
$$

where $l=1, \ldots, k$, and $|\alpha| \leq(d+1) l(M)$ (thus, the dimension $\mu$ equals $k \cdot r$ times the number of monomials in $r$ variables of degree $\leq(d+1) l(M))$. This mapping is injective for $|y|<\delta^{\prime}$ in view of Theorem 2.1 and Assertion 3.1.1. Consequently, we have a smooth injective mapping from a neighborhood of the origin in $\mathbb{R}^{m}$ into $\mathbb{R}^{\mu}$. This implies that $m \leq \mu$ and hence the desired finite dimensionality of the conclusion of Theorem 2 .

\subsection{The non-minimal case.}

We shall prove a generalization of Theorem 2 for the case where $M$ is not minimal. Before stating this result, we need some notation. Let $M \subset \mathbb{C}^{N}$ be a real analytic CR submanifold, and let $p_{0} \in M$. Denote by $\mathcal{F}_{M}\left(p_{0}\right)$ the ring of germs of $C^{\infty}$ real valued functions on $M$ at $p_{0}$ which are also CR, and by $\mathcal{G}_{M}\left(p_{0}\right)$ the subring consisting of those that are real analytic, i.e. those that are restrictions of the elements in $\mathcal{O}_{N}\left(p_{0}\right)$ which are real valued on $M$. If $f$ is a representative of an element in $\mathcal{F}_{M}\left(p_{0}\right)$ then the restriction of $f$ to each CR orbit is constant. (Conversely, if $f$ is a real-valued $C^{\infty}$ function near $p_{0} \in M$ which is constant on the CR orbits then $f \in \mathcal{F}_{M}\left(p_{0}\right)$.) Hence, if $M$ is connected and minimal somewhere then $\mathcal{G}_{M}\left(p_{0}\right)=\mathcal{F}_{M}\left(p_{0}\right)=\mathbb{R}$. On the other hand, if $M$ is non-minimal everywhere then it follows from the Frobenius theorem that $\mathcal{G}_{M}(p)$ contains a non-trivial element if the CR orbit of $p$ has maximal dimension.

It is also easy to verify that $\operatorname{aut}(M, p)$ is a $\mathcal{F}_{M}(p)$-module, and $\operatorname{hol}(M, p)$ is a $\mathcal{G}_{M}(p)$-module, for every $p \in M$. Indeed, it is obvious that $\operatorname{hol}(M, p)$ 
is a $\mathcal{G}_{M}(p)$-module, and the fact that aut $(M, p)$ is a $\mathcal{F}_{M}(p)$-module is also immediate from the following characterization of aut $(M, p)$ (see e.g. [BR2]): a smooth real vector field $X$ on $M$ near $p \in M$ is in aut $(M, p)$ if and only if

$$
[\mathbb{L}, X] \in \mathbb{L},
$$

where $\mathbb{L}$ denotes the space of smooth $C R$ vector fields on $M$ near $p$.

The following is a generalization of Theorem 2 .

Theorem 3.2.1. Let $M \subset \mathbb{C}^{N}$ be a real analytic, holomorphically nondegenerate CR submanifold, and assume that $M$ is everywhere non-minimal. Then there is a dense open subset $\Omega_{a} \subset M$ such that aut $(M, p)$ is a finitely generated free $\mathcal{F}_{M}(p)$-module for $p \in \Omega_{a}$, and a dense open subset $\Omega_{h} \subset M$ such that $\operatorname{hol}(M, p)$ is a finitely generated free $\mathcal{G}_{M}(p)$-module for $p \in \Omega_{h}$.

We begin with a local description of generic manifolds which are everywhere non-minimal.

Proposition 3.2.2. Let $M \subset \mathbb{C}^{N}$ be a generic, real analytic submanifold of codimension $d$ which is everywhere non-minimal, and let $p_{0} \in M$ whose local $C R$ orbit is of maximal dimension. Then there are coordinates $\left(z, w^{\prime}, w^{\prime \prime}\right) \in \mathbb{C}^{n} \times \mathbb{C}^{d-q} \times \mathbb{C}^{q}=\mathbb{C}^{N}$, where $q$ denotes the codimension of the local $C R$ orbit of $p_{0}$ in $M$, vanishing at $p_{0}$ such that $M$ is defined by the equations

$$
\left\{\begin{array}{l}
\operatorname{Im} w^{\prime}=\phi\left(z, \bar{z}, \operatorname{Re} w^{\prime}, \operatorname{Re} w^{\prime \prime}\right) \\
\operatorname{Im} w^{\prime \prime}=0 ;
\end{array}\right.
$$

here, $\phi$ is a real valued analytic function with $\phi\left(z, 0, s^{\prime}, s^{\prime \prime}\right) \equiv 0$. Moreover, the local $C R$ orbit of the point $\left(z, w^{\prime}, w^{\prime \prime}\right)=\left(0,0, s^{\prime \prime}\right)$, for $s^{\prime \prime} \in \mathbb{R}^{q}$, is given by

$$
\left\{\begin{array}{l}
\operatorname{Im} w^{\prime}=\phi\left(z, \bar{z}, \operatorname{Re} w^{\prime}, s^{\prime \prime}\right) \\
w^{\prime \prime}=s^{\prime \prime}
\end{array}\right.
$$

Proof. Since the local CR orbit of $p_{0} \in M$ has maximal dimension, it follows from the Frobenius theorem that there are $h_{1}, \ldots, h_{q} \in \mathcal{O}_{N}\left(p_{0}\right)$ such that

$$
\partial h_{1} \wedge \ldots \wedge \partial h_{q} \neq 0
$$

near $p_{0}$, the restriction of each $h_{j}$ to $M$ is real valued, and such that the CR orbit of any point $p_{1}$ near $p_{0}$ is given by $\left\{p \in M h_{1}(p)=h_{1}\left(p_{1}\right), \ldots, h_{q}(p)=\right.$ 
$\left.h_{q}\left(p_{1}\right)\right\}$. Thus, there are coordinates $\left(Z^{\prime}, w^{\prime \prime}\right) \in \mathbb{C}^{n+d-q} \times \mathbb{C}^{q}$, vanishing at $p_{0}$, such $M$ is contained in $\operatorname{Im} w^{\prime \prime}=0$ and such that the CR orbits on $M$ are given as the intersections between $M$ and $w^{\prime \prime}=s^{\prime \prime}$, for $s^{\prime \prime}$ near $0 \in \mathbb{R}^{q}$. Since $M$ is generic and contained in the flat surface $\operatorname{Im} w^{\prime \prime}=0$, we can make a change of coordinates $\tilde{Z}^{\prime}=A\left(w^{\prime \prime}\right) Z^{\prime}$, where $A\left(w^{\prime \prime}\right)$ is an invertible $(n+d-q) \times(n+d-q)$-matrix with holomorphic matrix elements in $w^{\prime \prime}$, and write $\tilde{Z}^{\prime}=(u, v) \in \mathbb{C}^{n} \times \mathbb{C}^{d-q}$ such that $M$ is given by

$$
\left\{\begin{array}{l}
\operatorname{Im} v=\psi\left(u, \bar{u}, \operatorname{Re} v, \operatorname{Re} w^{\prime \prime}\right) \\
\operatorname{Im} w^{\prime \prime}=0,
\end{array} .\right.
$$

where $\psi\left(u, \bar{u}, s^{\prime}, s^{\prime \prime}\right)$ is real valued, real analytic, and satisfies

$$
\psi\left(0,0,0, s^{\prime \prime}\right) \equiv \frac{\partial \psi}{\partial u}\left(0,0,0, s^{\prime \prime}\right) \equiv \frac{\partial \psi}{\partial s^{\prime}}\left(0,0,0, s^{\prime \prime}\right) \equiv 0
$$

for all $s^{\prime \prime} \in \mathbb{R}^{q}$ near 0 . Now, we claim that we can actually find holomorphic coordinates $\left(z, w^{\prime}\right) \in \mathbb{C}^{n} \times \mathbb{C}^{d-q}$ of the form

$$
\left\{\begin{array}{l}
z=u \\
w^{\prime}=f\left(u, v, w^{\prime \prime}\right)
\end{array}\right.
$$

such that $M$ is defined by

$$
\left\{\begin{array}{l}
\operatorname{Im} w^{\prime}=\phi\left(z, \bar{z}, \operatorname{Re} w^{\prime}, \operatorname{Re} w^{\prime \prime}\right) \\
\operatorname{Im} w^{\prime \prime}=0,
\end{array}\right.
$$

where $\phi\left(z, \bar{z}, s^{\prime}, s^{\prime \prime}\right)$ is real valued, real analytic, and satisfies

$$
\phi\left(z, 0, s^{\prime}, s^{\prime \prime}\right) \equiv 0 .
$$

This follows from [BJT, Lemma 1.1] in the following way. Consider only the equation for $\operatorname{Im} v$ in (3.2.5) for a fixed $s^{\prime \prime}=\operatorname{Re} w^{\prime \prime}$. Lemma 1.1 in [BJT] asserts that there is a change of coordinates

$$
\left\{\begin{array}{l}
z=u \\
w^{\prime}=f\left(u, v ; s^{\prime \prime}\right),
\end{array}\right.
$$

holomorphic in $(u, v)$, such that the equation for $\operatorname{Im} v$ in (3.2.5) becomes the equation for $\operatorname{Im} w^{\prime}$ in (3.2.8) and $\phi$ is as in (3.2.9). Moreover, the change of coordinates is obtained by the implicit function theorem, so if we now think of $s^{\prime \prime}$ as a real analytic parameter in (3.2.5) we find that the change 
of coordinates (3.2.10) is real analytic in $s^{\prime \prime}$. Hence, it can be extended as a holomorphic change of coordinates of the form (3.2.7). This change of coordinates coincides with (3.2.10) on $\operatorname{Im} w^{\prime \prime}=0$, and since $M$ is contained in $\operatorname{Im} w^{\prime \prime}=0$ the claim is proved. This completes the proof of the proposition.

The following is an easy consequence of Proposition 3.2.2 and the definition of $k$-nondegeneracy.

Corollary 3.2.3. Let $M \subset \mathbb{C}^{N}$ be a real analytic, holomorphically nondegenerate $C R$ submanifold and $p \in M$. If the local $C R$ orbit $W_{p}$ of $p$ is of maximal dimension then, for any integer $k, M$ is $k$-nondegenerate at $p$ if and only if $W_{p}$ is $k$-nondegenerate at $p$. In particular, $l(M)=l\left(W_{p}\right)$.

Proof of Theorem 3.2.1. We start by showing that for $p$ in an open dense subset $\Omega_{a} \subset M$, aut $(M, p)$ is a finitely generated free $\mathcal{F}_{M}(p)$-module. Denote by $W_{p}$ the local CR orbit of any point $p \in M$. Let $\Omega_{a}^{1}$ be the dense open subset of points $p \in M$ such that the dimension of $W_{p}$ is maximal and such that $M$ is $l(M)$-nondegenerate at $p$. By Corollary 3.2.3, the CR orbit $W_{p}$, for $p \in \Omega_{a}^{1}$, is also $l(M)$-nondegenerate at $p$ and $l\left(W_{p}\right)=l(M)$. Next, define the following function on $\Omega_{a}^{1}$

$$
\alpha(p)=\operatorname{dim}_{\mathbb{R}}\left(\left.\operatorname{aut}(M, p)\right|_{p} / \tilde{T}_{p}\left(W_{p}\right)\right),
$$

where aut $\left.(M, p)\right|_{p}$ denotes the subspace of $T_{p}(M)$ obtained as values of the vector fields in aut $(M, p)$ and $\tilde{T}_{p}\left(W_{p}\right)=\left.\operatorname{aut}(M, p)\right|_{p} \cap T_{p}\left(W_{p}\right)$. Let $\Omega_{a}^{2}$ be the set of points $p \in \Omega_{a}^{1}$ such that both $\operatorname{dim}_{\mathbb{R}}$ aut $\left.(M, p)\right|_{p}$ and $\alpha(p)$ are maximal in a neighborhood of $p$. Using the fact that the the CR orbits $W_{q}$ for $q$ in a neighborhood of $p \in \Omega_{a}^{1}$ form a real analytic foliation of $M$, one can check by elementary linear algebra that $\Omega_{a}^{2}$ is open and dense in $\Omega_{a}^{1}$ and that $\alpha(q)$ is constant for $q$ in a neighborhood of $p$.

Let us denote by aut $(M, p)^{\prime}$ the subspace of aut $(M, p)$ consisting of those vector fields that are also tangent to $W_{q}$ for all $q$ in a neighborhood of $p$. It follows immediately from (3.2.1) that the restriction of any $X \in$ aut $(M, p)^{\prime}$ to $W_{q}$, for $q$ in a neighborhood of $p$, is in aut $\left(W_{q}, q\right)$. We define the following function in $\Omega_{a}^{1}$

$$
\beta(p)=\left.\operatorname{dim}_{\mathbb{R}} \operatorname{aut}(M, p)^{\prime}\right|_{W_{p}},
$$

where aut $\left.(M, p)^{\prime}\right|_{W_{p}}$ denotes the subspace of aut $\left(W_{p}, p\right)$ obtained by taking restrictions to $W_{p}$ of the vector fields in aut $(M, p)^{\prime}$. Let $d$ denote the codi- 
mension of the maximal $\mathrm{CR}$ orbits in $\mathbb{C}^{N}$. It follows from Theorem 1 and Corollary 3.2.3 that $0 \leq \beta(p) \leq \mu$, where $\mu$ denotes the $\operatorname{dim}_{\mathbb{R}} M$ times the number of monomials in $\operatorname{dim}_{\mathbb{R}} M$ variables of degree $\leq(d+1) l(M)$, for all $p \in \Omega_{a}^{1}$. Clearly, if we have vector fields $Y_{1}, \ldots, Y_{k}$ on $M$ that are tangent to each $W_{q}$, for $q$ near $p \in \Omega_{a}^{1}$, and linearly independent over $\mathbb{R}$ as vector fields on $W_{p}$ then they are also linearly independent over $\mathbb{R}$ on $W_{q}$, for all $q$ in a neighborhood of $p$. We let $\Omega_{a}^{3}$ be the set of points $p \in \Omega_{a}^{1}$ for which $\beta(p)$ is maximal in a neighborhood of $p$. It follows easily that $\Omega_{a}^{3}$ is a dense open subset of $\Omega_{a}^{1}$ and that $\beta(q)$ is constant for $q$ in a neighborhood of $p$.

Now, let $\Omega_{a}$ be the intersection of $\Omega_{a}^{2}$ and $\Omega_{a}^{3}$ in $\Omega_{a}^{1}$. This is a dense open subset of $M$, and we claim that aut $\left(M, p_{0}\right)$ is a finitely generated free $\mathcal{F}_{M}\left(p_{0}\right)$-module for every $p_{0} \in \Omega_{a}$. Let $X_{1}^{\prime}, \ldots, X_{\beta\left(p_{0}\right)}^{\prime}, T_{1}, \ldots, T_{\alpha\left(p_{0}\right)} \in$ $\operatorname{aut}\left(M, p_{0}\right)$ be such that the images of $X_{1}^{\prime}, \ldots, X_{\beta\left(p_{0}\right)}^{\prime}$ in $\left.\operatorname{aut}\left(M, p_{0}\right)^{\prime}\right|_{W_{p_{0}}}$ form a basis for that space, and the images of $T_{1}, \ldots, T_{\alpha\left(p_{0}\right)}$ in $\left.\operatorname{aut}\left(M, p_{0}\right)\right|_{p_{0}} / \tilde{T}_{p_{0}}\left(W_{p_{0}}\right)$ form a basis for the latter. As we noted above, the images of these vector fields are linearly independent in the corresponding vector spaces at every $p$ in a neighborhood of $p_{0}$. This implies that these vector fields are linearly independent over the ring $\mathcal{F}_{M}\left(p_{0}\right)$. To see this, assume that there are $c_{1}, \ldots, c_{\alpha\left(p_{0}\right)}, d_{1}, \ldots, d_{\beta\left(p_{0}\right)} \in \mathcal{F}_{M}\left(p_{0}\right)$ such that

$$
\sum_{i=1}^{\beta\left(p_{0}\right)} d_{i} X_{i}^{\prime}+\sum_{j=1}^{\alpha\left(p_{0}\right)} c_{j} T_{j} \equiv 0
$$

Taking the image of (3.2.13) in aut $\left.(M, p)\right|_{p} / \tilde{T}_{p}\left(W_{p}\right)$, for $p$ near $p_{0}$, and using the fact that the $X_{i}^{\prime}$ are tangent to $W_{p}$, we deduce that $c_{1} \equiv \ldots \equiv c_{\alpha\left(p_{0}\right)} \equiv 0$. Then taking the image in aut $\left.(M, p)\right|_{W_{p}}$ and using the fact that the $d_{i}$ are constant on $W_{p}$, we deduce that $d_{1} \equiv \ldots \equiv d_{\beta\left(p_{0}\right)} \equiv 0$. Hence, the vector fields are linearly independent over $\mathcal{F}_{M}\left(p_{0}\right)$.

It remains to prove that these vector fields generate aut $\left(M, p_{0}\right)$ as a $\mathcal{F}_{M}\left(p_{0}\right)$-module. Now, since both $\alpha(p)$ and $\beta(p)$ are constant in a neighborhood of $p_{0}$, it follows that the images of $X_{1}^{\prime}, \ldots, X_{\beta\left(p_{0}\right)}^{\prime}$ in aut $\left.(M, p)^{\prime}\right|_{W_{p}}$ form a basis for this vector space for all $p$ in a neighborhood of $p_{0}$, and similarly for the images of $T_{1}, \ldots, T_{\alpha\left(p_{0}\right)}$. Hence, the vector spaces aut $\left.(M, p)^{\prime}\right|_{W_{p}}$ and $\left.\operatorname{aut}(M, p)\right|_{p} / \tilde{T}_{p}\left(W_{p}\right)$ form $C^{\infty}$ real vector bundles (not real analytic since the coefficients of these vector fields are merely $C^{\infty}$ functions) over $M$ in a neighborhood of $p_{0}$. Thus, if we take any $X \in \operatorname{aut}\left(M, p_{0}\right)$ and consider its image in the latter vector bundle we obtain smooth real valued functions 
$c_{1}, \ldots, c_{\alpha\left(p_{0}\right)}$ on $M$ such that

$$
X^{\prime}=X-\sum_{j=1}^{\alpha\left(p_{0}\right)} c_{j} T_{j}
$$

is tangent to $W_{p}$ for all $p$ in a neighborhood of $p_{0}$. We claim that

$$
\begin{aligned}
& X^{\prime} \in \operatorname{aut}\left(M, p_{0}\right)^{\prime} \\
& c_{1}, \ldots, c_{\alpha\left(p_{0}\right)} \in \mathcal{F}_{M}\left(p_{0}\right) .
\end{aligned}
$$

To verify this, we compute the bracket of $X$ with a $\mathrm{CR}$ vector field $L \in \mathbb{L}$

$$
\begin{aligned}
{[L, X] } & =\left[L, X^{\prime}\right]+\sum_{j=1}^{\alpha\left(p_{0}\right)}\left[L, c_{j} T_{j}\right] \\
& =\left[L, X^{\prime}\right]+\sum_{j=1}^{\alpha\left(p_{0}\right)} c_{j}\left[L, T_{j}\right]+\left(L c_{j}\right) T_{j} .
\end{aligned}
$$

Since $X, T_{1}, \ldots, T_{\alpha\left(p_{0}\right)} \in \operatorname{aut}(M, p)$, we know that

$$
[L, X],\left[L, T_{1}\right], \ldots,\left[L, T_{\alpha\left(p_{0}\right)}\right] \in \mathbb{L} .
$$

Also, since both $X^{\prime}$ and $L$ are tangent to $W_{p}$ for all $p$ near $p_{0},(3.2 .17)$ implies that

$$
\sum_{j=1}^{\alpha\left(p_{0}\right)}\left(L c_{j}\right) T_{j}
$$

is tangent to $W_{p}$ for all $p$ near $p_{0}$. Since the images of $T_{1}, \ldots, T_{\alpha\left(p_{0}\right)}$ in the vector space $\left.\operatorname{aut}(M, p)\right|_{p} / \tilde{T}_{p}\left(W_{p}\right)$ are linearly independent for $p$ near $p_{0}$, we deduce that $L c_{1}(p), \ldots, L c_{\alpha\left(p_{0}\right)}(p)=0$ for all $p$ in a neighborhood of $p_{0}$. Hence, (3.2.16) is proved, because $L \in \mathbb{L}$ was arbitrary. Moreover, (3.2.15) also follows because now (3.2.1) implies that $\left[L, X^{\prime}\right] \in \mathbb{L}$. The claim is proved.

To finish the proof of the first part of the theorem, we have to prove that $X^{\prime} \in \operatorname{aut}\left(M, p_{0}\right)^{\prime}$ can be written

$$
X^{\prime}=\sum_{i=1}^{\beta\left(p_{0}\right)} d_{j} X_{j}^{\prime},
$$


where $d_{1}, \ldots, d_{\beta\left(p_{0}\right)} \in \mathcal{F}_{M}\left(p_{0}\right)$. By taking the image in the real $C^{\infty}$ vector bundle aut $\left.(M, p)^{\prime}\right|_{W_{p}}$, we obtain smooth real valued functions $d_{1}, \ldots, d_{\beta\left(p_{0}\right)}$ such that (3.2.19) holds. Since the values $d_{1}, \ldots, d_{\beta\left(p_{0}\right)}$ are unique as real numbers on each CR orbit, it follows that each function $d_{j}(p)$ is constant on the CR orbits near $p_{0}$ and, hence, $d_{j} \in \mathcal{F}_{M}\left(p_{0}\right)$. This completes the proof of the statement that aut $\left(M, p_{0}\right)$ is a finitely generated free $\mathcal{F}_{M}\left(p_{0}\right)$-module at every $p_{0} \in \Omega_{a}$.

To prove the corresponding statement for $\operatorname{hol}(M, p)$ we define $\Omega_{h}$ in complete analogy with $\Omega_{a}$, replacing aut $(M, p)$ by $\operatorname{hol}(M, p)$. The same proof as above, mutatis mutandi, completes the proof of the statement that $\operatorname{hol}\left(M, p_{0}\right)$ is a finitely generated free $\mathcal{G}_{M}\left(p_{0}\right)$-module, since the vector bundles corresponding to those with fibers aut $\left.(M, p)\right|_{p} / T_{p}\left(W_{p}\right)$ and $\left.\operatorname{aut}(M, p)^{\prime}\right|_{W_{p}}$, replacing aut $(M, p)$ by $\operatorname{hol}(M, p)$, are real analytic. We leave the details of this to the reader.

\section{Proof of Theorem 3 and examples.}

\subsection{Proof of Theorem 3.}

To prove Theorem 3 , suppose first that $M$ is holomorphically degenerate. Then for any $p \in M$ there is a germ of a vector field $X=\sum_{j=1}^{N} c_{j}(Z) \frac{\partial}{\partial Z_{j}}$, with $c_{j}(Z)$ holomorphic, tangent to $M$ near $p$ with nonzero restriction to $M$. Let $h(Z)$ be any holomorphic function nontrivial on $M$ with $h(p)=0$ and $K$ a positive integer as in the statement of the theorem. Then the vector field $Y=\operatorname{Re}\left(h(Z)^{K} X\right)$ is a nontrivial element of $\operatorname{hol}_{\mathbb{R}}\left(M, p_{0}\right)$ and vanishes to order at least $K$ at $p$. For $t>0$ small the CR mapping $F=\exp t Y$ extends to a local biholomorphism mapping $M$ into itself with $F(p)=p$. It is not hard to check that all derivatives of $F$ up to order $K$ agree with those of the identity mapping, $I$, yet $F \not \equiv I$ on $M$. This proves (0.4) with $G=I$.

Next, we show that $\operatorname{hol}_{\mathbb{R}}(M, p)$ is infinite dimensional whenever $M$ is holomorphically degenerate, or everywhere non-minimal and homogeneous. The proof of this in the case where $M$ is holomorphically degenerate is exactly the same as in the hypersurface case (see [St2]). If $M$ is homogeneous and everywhere non-minimal then there is a holomorphic polynomial $h(z, w)$ which is real valued and non-constant on $M$ (see [BER1]). Also, there is a non-trivial vector field $X \in \operatorname{hol}(M, p)$ (e.g. the infinitesimal dilation; see [St1]). The infinite dimensionality of $\operatorname{hol}(M, p)$ follows by noting that 
$h^{k} X \in \operatorname{hol}\left(M, p_{0}\right)$, for $k=0,1, \ldots$, are all linearly independent over $\mathbb{R}$. We note also that by considering the mappings $\exp \left(t h(z, w)^{K} X\right)$, we can construct nontrivial local biholomorphisms which agree with the identity up to any preassigned order.

Finally, we shall show that if $M$ is not minimal almost everywhere then for $p \in M$ outside an open dense subset if $\operatorname{dim}_{\mathbb{R}} \operatorname{hol}(M, p) \neq 0$, then $\operatorname{dim}_{\mathbb{R}} \operatorname{hol}(M, p)=\infty$. Indeed, suppose the local CR orbit of $p$ is of maximal dimension. Then it follows from Proposition 3.2.2 that there is a holomorphic function $h(Z)$ whose restriction to $M$ is real and nonconstant. If $X$ is a nontrivial vector field in $\operatorname{hol}(M, p)$, then so is $X_{k}=h(Z)^{k} X$ for any positive integer $k$. Since the $X_{k}$ are all linearly independent as vector fields over $\mathbb{R}$, it follows that $\operatorname{dim}_{\mathbb{R}} \operatorname{hol}(M, p)=\infty$. This completes the proof of Theorem 3 .

\subsection{A holomorphically nondegenerate, nowhere minimal CR submanifold with $\operatorname{hol}(M, 0)=\{0\}$.}

In Theorem 3, it is shown that for most points $p \in M$, if $\operatorname{hol}(M, p)$ contains at least one non-trivial element and if $M$ is nowhere minimal, then $\operatorname{dim}_{\mathbb{R}} \operatorname{hol}(M, p)=\infty$. In this section we construct an example to show it may happen that $\operatorname{hol}(M, p)=\{0\}$ for a holomorphically nondegenerate, nowhere minimal CR submanifold $M$.

N. Stanton [St3] has given examples of real hypersurfaces with no (nontrivial) infinitesimal $\mathrm{CR}$ automorphisms. We leave it to the reader to verify that a slight modification of the argument of [St3] proves the following.

Proposition 4.2.1 ([St3]). Let $M^{0} \subset \mathbb{C}^{2}$ be the hypersurface defined by

$$
\operatorname{Im} w=z^{4} \bar{z}^{10}+z^{10} \bar{z}^{4}+(\operatorname{Re} w)|z|^{8} .
$$

Then $\operatorname{aut}\left(M^{0}, 0\right)=\{0\}$ (and hence also hol $(M, 0)=\{0\}$ ).

We will use this to prove:

Proposition 4.2.2. Let $M \subset \mathbb{C}^{3}$ be defined by

$$
\left\{\begin{array}{l}
\operatorname{Im} w_{1}=z^{4} \bar{z}^{10}+z^{10} \bar{z}^{4}+\left(\operatorname{Re} w_{1}\right)|z|^{8}+\left(\operatorname{Re} w_{2}\right)|z|^{4} \\
\operatorname{Im} w_{2}=0 .
\end{array}\right.
$$

Then $\operatorname{hol}(M, 0)=\{0\}$. 
Remark. Note that $M$ is holomorphically nondegenerate and nowhere minimal.

Proof. Assume that

$$
\begin{aligned}
X= & a(z, w) \frac{\partial}{\partial z}+\bar{a}(\bar{z}, \bar{w}) \frac{\partial}{\partial \bar{z}}+b(z, w) \frac{\partial}{\partial w_{1}} \\
& +\bar{b}(\bar{z}, \bar{w}) \frac{\partial}{\partial \bar{w}_{1}}+c(z, w) \frac{\partial}{\partial w_{2}}+\bar{c}(\bar{z}, \bar{w}) \frac{\partial}{\partial \bar{w}_{2}}
\end{aligned}
$$

is in $\operatorname{hol}(M, 0)$. This is the same as saying that the holomorphic vector field

$$
\begin{aligned}
Y= & a(z, w) \frac{\partial}{\partial z}+\bar{a}(\chi, \tau) \frac{\partial}{\partial \chi}+b(z, w) \frac{\partial}{\partial w_{1}} \\
& +\bar{b}(\chi, \tau) \frac{\partial}{\partial \tau_{1}}+c(z, w) \frac{\partial}{\partial w_{2}}+\bar{c}(\chi, \tau) \frac{\partial}{\partial \tau_{2}}
\end{aligned}
$$

in $\mathbb{C}^{6}$ is tangent to the complexification $\mathcal{M}$ of $M$ in $\mathbb{C}^{6}$

$$
\left\{\begin{array}{l}
w_{1}-\tau_{1}-2 i\left(z^{4} \chi^{10}+z^{10} \chi^{4}\right)-i\left(w_{1}+\tau_{1}\right) z^{4} \chi^{4}-i\left(w_{2}+\tau_{2}\right) z^{2} \chi^{2}=0 \\
w_{2}-\tau_{2}=0
\end{array}\right.
$$

Let us denote the first equation above by $\rho(z, w, \chi, \tau)=0$. It is an easy exercise to verify that if $Y$ is tangent to $\mathcal{M}$ then $b(z, w)$ and $c(z, w)$ are independent of $z$ and real, i.e. $b=\bar{b}$ and $c=\bar{c}$. We will use the notation $b=b(w)$ and $c=c(w)$. We let

$$
Y^{\prime}=a(z, w) \frac{\partial}{\partial z}+\bar{a}(\chi, \tau) \frac{\partial}{\partial \chi}+b(w) \frac{\partial}{\partial w_{1}}+\bar{b}(\tau) \frac{\partial}{\partial \tau_{1}}
$$

and

$$
\rho_{0}\left(z, w_{1}, \chi, \tau_{1}\right)=w_{1}-\tau_{1}-2 i\left(z^{4} \chi^{10}+z^{10} \chi^{4}\right)-i\left(w_{1}+\tau_{1}\right) z^{4} \chi^{4} .
$$

Note that $\rho_{0}=0$ is the defining equation of the complexification $\mathcal{M}^{0}$ of $M^{0}$ in $\mathbb{C}^{4}$. Applying $Y$ to $\rho$, we obtain

$$
\begin{aligned}
(Y \rho)(z, w, \chi, \tau)= & \left(Y^{\prime} \rho_{0}\right)(z, w, \chi, \tau)-2 i a(z, w)\left(w_{2}+\tau_{2}\right) z \chi^{2} \\
& -2 i \bar{a}(\chi, \tau)\left(w_{2}+\tau_{2}\right) z^{2} \chi-i(c(w)+c(\tau)) z^{2} \chi^{2}
\end{aligned}
$$

Since $Y$ is assumed tangent to $\mathcal{M}$, this expression is 0 on $\mathcal{M}$. We can solve for $\tau$ in the defining equations of $\mathcal{M}$ and obtain

$$
\tau_{1}=\bar{Q}_{0}\left(\chi, z, w_{1}\right)+O\left(w_{2}\right), \quad \tau_{2}=w_{2},
$$


where $\tau_{1}=\bar{Q}_{0}\left(\chi, z, w_{1}\right)$ is the defining equation of $\mathcal{M}^{0}$ in $\mathbb{C}^{4}$ and $O\left(w_{2}\right)$ denotes, as usual, terms that contain the factor $w_{2}$. We have also substituted $\tau_{2}=w_{2}$ in the first equation. Let us write (4.2.9) as $\tau=\bar{Q}(\chi, z, w)$ for short. Note that $\bar{Q}(0, z, w) \equiv \bar{Q}(\chi, 0, w) \equiv w$ and $\bar{Q}_{0}\left(0, z, w_{1}\right) \equiv \bar{Q}_{0}\left(\chi, 0, w_{1}\right) \equiv w_{1}$. Substituting in (4.2.8) we obtain

$$
\begin{aligned}
& \left(Y^{\prime} \rho_{0}\right)(z, w, \chi, \bar{Q}(\chi, z, w)) \equiv \\
& 4 i a(z, w) w_{2} z \chi^{2}+4 i \bar{a}(\chi, \bar{Q}(\chi, z, w)) w_{2} z^{2} \chi+i(c(w)+c(\bar{Q}(\chi, z, w))) z^{2} \chi^{2} .
\end{aligned}
$$

Let us expand the holomorphic vector field $Y^{\prime}\left(z, w_{1}, w_{2}, \chi, \tau_{1}, w_{2}\right)$ in $w_{2}$. We obtain

$$
Y^{\prime}\left(z, w_{1}, w_{2}, \chi, \tau_{1}, w_{2}\right)=\sum_{k=k_{0}}^{\infty} Y_{k}^{\prime}\left(z, w_{1}, \chi, \tau_{1}\right) w_{2}^{k},
$$

where each $Y_{k}^{\prime}$ is a holomorphic vector field in the variables $\left(z, w_{1}, \chi, \tau_{1}\right)$ and where in particular the vector field $Y_{k_{0}}^{\prime}$ is not identically 0 ; we assume here, in order to obtain a contradiction, that $Y^{\prime}$ is not identically 0 . Note that, since $k_{0}$ is assumed to be the lowest order in the expansion of $Y^{\prime}\left(z, w_{1}, w_{2}, \chi, \tau_{1}, w_{2}\right)$, the coefficients $a\left(z, w_{1}, w_{2}\right)$ and $b\left(w_{1}, w_{2}\right)$ have to be divisible by $w_{2}^{k_{0}}$ ( $k_{0}$ could, of course, be 0 ). We expand $a, b, c$ in $w_{2}$ as follows (4.2.12)

$$
a(z, w)=\sum_{k=k_{0}}^{\infty} a_{k}\left(z, w_{1}\right) w_{2}^{k}, b(w)=\sum_{k=k_{0}}^{\infty} b_{k}\left(w_{1}\right) w_{2}^{k}, c(w)=\sum_{k=0}^{\infty} c_{k}\left(w_{1}\right) w_{2}^{k} .
$$

Identifying the coefficients of the lowest order term in $w_{2}$ (i.e. of $w_{2}^{k_{0}}$ ) in (4.2.10), using the fact that

$$
\bar{Q}(\chi, z, w)=\left(\bar{Q}_{0}\left(\chi, z, w_{1}\right)+O\left(w_{2}\right), w_{2}\right),
$$

we find

$$
\left(Y_{k_{0}}^{\prime} \rho_{0}\right)\left(z, w_{1}, \chi, \bar{Q}_{0}\left(\chi, z, w_{1}\right)\right)=i\left(c_{k_{0}}\left(w_{1}\right)+c_{k_{0}}\left(Q_{0}\left(\chi, z, w_{1}\right)\right)\right) z^{2} \chi^{2} .
$$

Now, it is easy to see that the expansion of the left hand side in terms of $z$ and $\chi$ does not contain a term with $z^{2} \chi^{2}$. The expansion of the right hand side contains the term

$$
2 i c_{k_{0}}\left(w_{1}\right) z^{2} \chi^{2}
$$


Thus, we must have $c_{k_{0}}\left(w_{1}\right) \equiv 0$ and

$$
\left(Y_{k_{0}}^{\prime} \rho_{0}\right)\left(z, w_{1}, \chi, \bar{Q}_{0}\left(\chi, z, w_{1}\right)\right) \equiv 0 .
$$

The latter implies that the vector field $Y_{k_{0}}^{\prime}$ in $\mathbb{C}^{4}$ is tangent to $\mathcal{M}^{0}$ or, equivalently, that the vector field $X_{k_{0}}^{\prime}$ in $\mathbb{C}^{2}$, obtained by formally replacing $\chi$ by $\bar{z}$ and $\tau_{1}$ by $\bar{w}_{1}$ in $Y_{k_{0}}^{\prime}$, is tangent to $M^{0}$. Now, $X_{k_{0}}^{\prime}$ is the real part of a holomorphic vector field so, since $X_{k_{0}}^{\prime}$ is tangent to $M^{0}, X_{k_{0}}^{\prime} \in \operatorname{hol}\left(M^{0}, 0\right)$ and hence $X_{k_{0}}^{\prime} \equiv 0$ by Proposition 4.2.1. This contradicts the fact that $Y_{k_{0}}^{\prime}$ was assumed $\not \equiv 0$. Consequently, $Y^{\prime}$ is identically 0 . That means $Y$ has to be of the form

$$
c(w) \frac{\partial}{\partial w_{2}}+c(\tau) \frac{\partial}{\partial \tau_{2}}
$$

It is easy to check that this implies $c(w) \equiv 0$ as well. This completes the proof of Proposition 4.2.2.

\section{Remarks.}

We shall restrict our remarks to the case where $M$ is a generic manifold. For any $p \in M$ we let $G_{p}$ denote the set of germs $H$ of biholomorphisms near $p$, with $H(M) \subset M$ and $H(p)=p$. It is easy to see that the set $G_{p}$ forms a group under composition of mappings. We have the following.

Theorem 5.1. Let $M$ be a real analytic, holomorphically nondegenerate, generic submanifold of $\mathbb{C}^{N}$ which is minimal at some point. For all $p \in M$, there is a unique topology on the group $G_{p}$ with respect to which it is a Lie group whose Lie algebra is $\operatorname{hol}(M, p)$.

This theorem follows from a slight modification of the proof of Theorem 3.1 of Kobayashi [K, p.13], by making use of Theorem 2. Indeed, if $\operatorname{dim}_{\mathbb{R}} \operatorname{hol}(M, p)$ is finite, the exponential of $\operatorname{hol}_{0}(M, p)$ (those vector fields in $\operatorname{hol}(M, p)$ that vanish at $p)$ is a connected Lie group $G_{p}^{0}$ which is a normal subgroup of $G_{p}$. (The vector fields in $\operatorname{hol}_{0}(M, p)$ can be integrated for all time in the sense of germs.) One may then impose the unique topology on $G_{p}$ for which $G_{p} / G_{p}^{0}$ is discrete. However, there is a natural topology for the group $G_{p}$ obtained by regarding $G_{p}$ as a subspace of the space of holomorphic mappings. Also, using Corollary of $\S 0$, one can embed $G_{p}$ as a subgroup of the group of invertible $(d+1) l(M)$-jets from which it inherits 
a topology. In general one does not know if these three topologies coincide. This question will be addressed in future work [BER2].

\section{References.}

[BER1] M.S. Baouendi, P. Ebenfelt and L.P. Rothschild, Algebraicity of holomorphic mappings between real algebraic sets in $\mathbb{C}^{n}$, Acta Math. 177 (1996), 225-273.

[BER2] , The local automorphism group of a real analytic hypersurface, Asian J. Math. 1 (1997). 1-16.

[BJT] M.S. Baouendi, H. Jacobowitz and F. Treves, On the analyticity of CR mappings, Ann. of Math. 122 (1985), 365-400.

[BR1] M.S. Baouendi and L.P. Rothschild, Holomorphic mappings between algebraic hypersurfaces in complex space, in Séminaire "Equations aux derivées partielles" 1994-1995, Ecole Polytechnique, Palaiseau, France, 1994.

[BR2] Transversal Lie group actions on abstract CR manifolds, Math. Ann. 287 (1990), 19-33.

[BR3] Mappings of real algebraic hypersurfaces, J. Amer. Math. Soc. 8 (1995), 997-1015.

[Be] V.K. Beloshapka, On the dimension of the group of automorphisms of an analytic hypersurface, Math. USSR Izv. 14 (1980), 223-245.

[BG] T. Bloom and I. Graham, On type conditions for generic real submanifolds of $\mathbb{C}^{n}$, Invent. Math. 40 (1977), 217-243.

[K] S. Kobayashi, Transformation groups in differential geometry, Springer Verlag, Berlin 1972.

[L] A.V. Loboda, On local automorphisms of real-analytic hypersurfaces, Math. USSR Izv. 18 (1982), 537-559.

[St1] N. Stanton, Infinitesimal CR automorphisms of rigid hypersurfaces, Amer. J. Math. 117 (1995), 141-16.

[St2] Infinitesimal CR automorphisms of real hypersurfaces, Amer. J. Math. 118 (1996), 209-233.

[St3] Real hypersurfaces with no infinitesimal CR automorphisms, preprint, 1995. 
[Ta] N. Tanaka, On the pseudo-conformal geometry of hypersurfaces of the space of $n$ complex variables, J. Math. Soc. Japan, 14 (1962), 397-429.

[Tu1] A.E. Tumanov, Extending CR functions on manifolds of finite type to a wedge, Mat. Sbornik, 136 (1988), 128-139.

[Tu2] Finite-dimensionality of the group of $C R$ automorphisms of a standard CR manifold, and proper holomorphic mappings of Siegel domains, Izvestia Akad. Nauk SSSR, Ser. Mat. 52 (1988); English transl. in Math. USSR Izvestia 32 (1989), 655-662.

[TH] A.E. Tumanov and G.M. Henkin, Local characterization of holomorphic automorphisms of Siegel domains, Funktsional. Anal. i Prilozhen. 17 (1983), 49-61; English transl. in Functional Anal. Appl. 17 (1983).

ReCeived March 14, 1996.

University of California at SAN Diego

LA Jolla, CA 92093

AND

Royal Institute of TeChNOLOGY

10044 Stockholm, Sweden

E-mail addresses: SBAOUENDI@UCSD.EDU, EBENFELT@MATH.KTH.SE, LROTHSCHILD@UCSD.EDU 